\title{
Two-Component Fractional Quantum Hall Effect in the Half-Filled Lowest Landau Level in an Asymmetric Wide Quantum Well
}

\author{
N. Thiebaut ${ }^{1}$, M. O. Goerbig ${ }^{1}$ and N. Regnault ${ }^{2,3}$ \\ ${ }^{1}$ Laboratoire de Physique des Solides, Universite Paris-Sud, \\ CNRS UMR 8502, F-91405 Orsay Cedex, France \\ ${ }^{2}$ Laboratoire Pierre Aigrain, Departement de Physique, \\ ENS, CNRS, 24 rue Lhomond, 75005 Paris, France \\ ${ }^{3}$ Department of Physics, Princeton University, Princeton, New Jersey 08544, USA
}

(Dated: September 10, 2018)

\begin{abstract}
We investigate theoretically the fractional quantum Hall effect at half-filling in the lowest Landau level observed in asymmetric wide quantum wells. The asymmetry can be achieved by a potential bias applied between the two sides of the well. Within exact-diagonalization calculations in the spherical geometry, we find that the incompressible state is described in terms of a two-component wave function. Its overlap with the ground state can be optimized with the help of a rotation in the space of the pseudospin, which mimics the lowest two electronic subbands.
\end{abstract}

\section{INTRODUCTION}

The study of the fractional quantum Hall effect (FQHE) is intimitely bound to progress in the fabrication of high-mobility samples, and the highest mobilities are achieved today in wide GaAs/AlGaAs quantum wells. A drawback of a large well width is, however, the stronger three-dimensional character of the electron motion, whereas the FQHE is a manifestation of electronic correlations in a strong magnetic field in a twodimensional (2D) electron system - strictly speaking, the $2 \mathrm{D}$ character is preserved in quantum wells only as long as the electronic-subband separation is larger than the other relevant energy scales, mainly the Coulomb interaction. In this case, important insight into the underlying electron liquids and their exotic excitations has been provided by studying trial wave functions ${ }^{1-4}$ for electrons in the lowest subband and in a single spin branch. The latter assumption was soon criticized by Halperin, who pointed out that the Zeeman effect is insufficient to justify this assumption because it is generally much lower than the leading interaction energy scale. To cure this drawback, he proposed 5 a two-component generalization of Laughlin's wave function ${ }^{1}$ that has proven to be extremely fruitful in the understanding of several twocomponent quantum Hall systems even if they are of lower symmetry than the original $\mathrm{SU}(2)$ spin symmetry. As an example beyond spin physics one may mention bilayer quantum Hall systems, ${ }^{6 / 7}$ where the layer index can be mimicked by a spin $1 / 2$, or more recently graphene with its internal spin-valley degree of freedom $\left[{ }^{8}\right.$

In order to understand the FQHE in wide quantum wells, while maintaining the $2 \mathrm{D}$ aspect of the system, only very recently a multi-component picture has been utilized, in which the different components consist of the electronic subbands. In the simplest case, one may restrict the theoretical description to the lowest two subbands, which are then mimicked by a pseudospin $s=$ $1 / 2{ }^{9}$ Within this model, exact-diagonalization ${ }^{9 / 10}$ and Monte-Carlo calculations 11 have shed light on a recently observed FQHE at a filling factor $\nu=n_{\mathrm{el}} / n_{B}=1 / 2$ (and $\nu=1 / 4), \frac{12 \mid 13}{1}$ where $n_{\mathrm{el}}$ is the electronic and $n_{B}$ the flux density. The states can be understood in terms of the so-called (331) [and (553)] Halperin wave function, the precise form of which is presented later in this paper [Eq. (3)]. In addition to the well width, wide quantum wells can be rendered asymmetric via a potential bias that is experimentally achieved by side gates and that presents a highly controllable parameter in the experimental study of the FQHE in these systems. Indeed, recent experiments revealed an intriguing transition from compressible to incompressible states when this potential bias is varied $13 \mid 14$

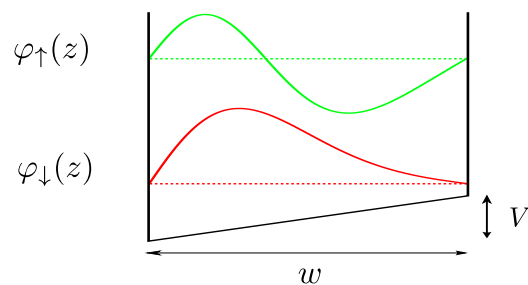

FIG. 1: (Color online) Scheme of the BQW, the first two eigenfunctions $\varphi_{\uparrow}$ and $\varphi_{\downarrow}$ are plotted.

Here we investigate the (331) Halperin state within a specially designed model for a biased quantum well (BQW) that consists of a wide quantum well with infinite potential walls. The potential bias is modeled by a linear slope $V z / w$, where $w$ is the well width in the $z$ direction (see Fig. 11). We perform exact-diagonalization studies of the Coulomb interaction within this model in the spherical geometry, where we take into account the two lowest electronic levels of the confinement potential that are mimicked by a pseudospin $s=1 / 2$. While at this stage the restriction to only two subbands is a model assumption, we justify it a posteriori by an investigation of the pseudospin polarization that indicates a preferential occupation of the lowest subband in the relevant parameter range. Our main finding is that the FQHE at $\nu=1 / 2$ in the lowest LL can be interpreted as a (331) Halperin 
state in a particular region of the $(w, V)$ parameter space, surrounded by compressible states. This result is in excellent agreement with recent experimental findings, $\frac{14115}{11}$ where a FQHE state at $\nu=1 / 2$ was stabilized in an intermediate bias range within a wide quantum well, while it vanishes for very small and large values of $V$. On a more technical side, our theoretical approach shows that the overlap of this state with the exact ground state can be largely enhanced at a variational parameter that physically plays the role of the pseudospin-polarization angle.

The paper is organized as follows. We introduce the two-band model of the biased wide quantum in the FQHE regime in Sec. [II. The subband polarization and the maximization of the overlap via the variational pseudospinpolarization angle are discussed in Sec. III] Section IV is devoted to a discussion of our main results, and we present our conclusions in Sec. V.

\section{MODEL}

When restricted to the lowest LL, the leading energy scale for the electronic degrees of freedom is set by the Coulomb interaction between electrons separated by the distance $r=\left|\mathbf{r}_{1}-\mathbf{r}_{2}\right|$ in the plane,

$$
\begin{aligned}
& V^{\sigma_{1} \ldots \sigma_{4}}(r) \\
& =\frac{e^{2}}{4 \pi \epsilon} \int_{0}^{w} \mathrm{~d} z \int_{0}^{w} \mathrm{~d} z^{\prime} \frac{\varphi_{\sigma_{1}}^{*}(z) \varphi_{\sigma_{2}}^{*}\left(z^{\prime}\right) \varphi_{\sigma_{3}}(z) \varphi_{\sigma_{4}}\left(z^{\prime}\right)}{\sqrt{r^{2}+\left(z-z^{\prime}\right)^{2}}},
\end{aligned}
$$

where $\varphi_{\sigma}(z)$ denotes the wave function in the $z$-direction associated with the first $(\sigma=\downarrow)$ and the second $(\sigma=\uparrow)$ subband of the confinement potential. The wave functions of the two lowest subbands are linear combinations of Airy functions of the first and the second kind. A second energy scale arises from the subband gap $\Delta(w, V)=$ $E_{2}-E_{1}$, which is itself a function of the parameters $w$ and $V$, and that may be obtained by solving the Schrödinger equation corresponding to the quantum mechanical problem of a particle in a the potential shown in Fig. 1. The subband gap plays the role of a Zeeman effect for the pseudospin. Notice furthermore that the third subband, which we neglect here, is found at an energy $E_{3}-E_{1} \sim 3 \Delta(w, V)$. In second quantized form, our model Hamiltonian reads

$$
\begin{array}{r}
H=\frac{1}{2} \sum_{\left\{m_{i}\right\}} \sum_{\left\{\sigma_{i}\right\}} V_{m_{1} \ldots m_{4}}^{\sigma_{1} \ldots \sigma_{4}}(w, V) c_{m_{1} \sigma_{1}}^{\dagger} c_{m_{2} \sigma_{2}}^{\dagger} c_{m_{4} \sigma_{4}} c_{m_{3} \sigma_{3}} \\
-\frac{\Delta(w, V)}{2} \sum_{m}\left(c_{m \uparrow}^{\dagger} c_{m \uparrow}-c_{m \downarrow}^{\dagger} c_{m \downarrow}\right),
\end{array}
$$

where the operators $c_{m, \sigma}^{(\dagger)}$ annihilate (create) an electron in the lowest-LL state $|n=0, m\rangle$ in the subband $\sigma$, where $m$ is the quantum number associated with the angular momentum. Here, we do not account for the physical spin degree of freedom and consider spinless fermions. A correct implementation of the physical spin, in addition to the subband pseudospin, would require a four-component treatment similarly to the case of graphene. $\frac{16117}{10}$ This would substantially reduce the number of particles that can be diagonalized. Instead, we rely on the fact that exchange effects generally favor spin-polarized states for a (spin) $\mathrm{SU}(2)$-symmetric interaction that are further stabilized by the Zeeman effect. The matrix elements $V_{m_{1} \ldots m_{4}}^{\sigma_{1} \ldots \sigma_{4}}(w, V)$ correspond to the two-body interaction (1) when projected to the lowest LL. A large number of non-zero matrix elements needs to be taken into account, in contrast to $\mathrm{SU}(2)$-symmetric spin systems or bilayer quantum Hall systems, where $\sigma_{1}=\sigma_{3}$ and $\sigma_{2}=\sigma_{4}$. Notice, however, that the latter (bilayer) approximation was made in Ref. 11, where the FQHE at half-filling was investigated via Monte-Carlo calculations. (a) $\left\langle S^{2}\right\rangle$

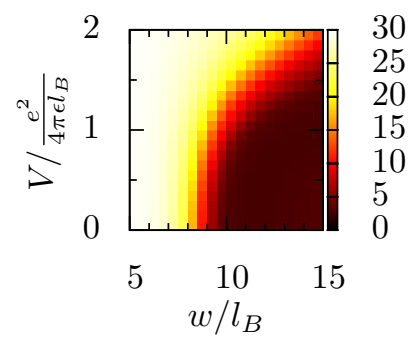

(b) $\left\langle S_{z}\right\rangle$

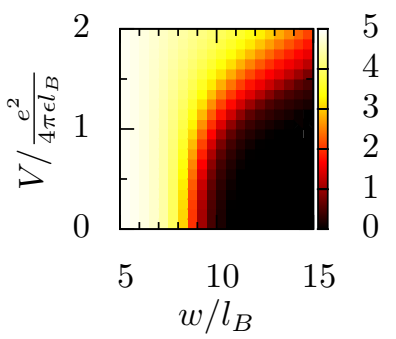

FIG. 2: (Color online) Norm and $z$-component of pseudospin for $N=10$ and $N_{B}=17$.

Within exact-diagonalization studies in the spherical geometry, we have investigated the ground-state properties at half-filling in the lowest LL for $N=6,8$, and 10 particles. The number of flux quanta threading the sphere is $N_{B}=2 N+\delta$, where $\delta$ is a state-dependent shift that is relevant in finite-size studies on the sphere. Here, we are interested in the Halperin (331) state ${ }^{5}$

$$
\begin{aligned}
\Psi_{(331)}\left(\left\{z_{i}^{\uparrow}\right\},\right. & \left.\left\{z_{i}^{\downarrow}\right\}\right)=\prod_{i<j \leq N / 2}\left(z_{i}^{\uparrow}-z_{j}^{\uparrow}\right)^{3} \\
& \times \prod_{i<j \leq N / 2}\left(z_{i}^{\downarrow}-z_{j}^{\downarrow}\right)^{3} \prod_{i, j \leq N / 2}\left(z_{i}^{\uparrow}-z_{j}^{\downarrow}\right) .
\end{aligned}
$$

which has a shift $\delta=-3 \sqrt{18}$ In Eq. (3), the complex positions $z_{j}^{\sigma}$ are those of particles in the pseudospin state $\sigma$, and we have omitted the ubiquitous Gaussian factor. The Hilbert space for 10 particles (and $N_{B}=17$ ) is of dimension $1.2 \times 10^{7}$ and the largest system size accessible numerically. In the remainder of the paper, we present our results only for 10 particles since the results for $N=6$ and 8 are qualitatively the same. We have concentrated our investigation on the experimentally relevant parameter range $w=5 \ldots 15 l_{B}$ and $V=0 \ldots 2 e^{2} / \epsilon l_{B}$, where the 
ground state is always found in the sector with a total angular momentum $L_{z}=0$ that indicates a homogeneous charge distribution, necessary for possible FQHE states. Here and in the following sections, we measure lengths in units of the magnetic length $l_{B} \simeq 25.8 \mathrm{~nm} / \sqrt{B[\mathrm{~T}]}$ and energies in units of the Coulomb energy $e^{2} / \epsilon l_{B}$, where $\epsilon$ is the dielectric constant of the host semiconductor material.

\section{SUBBAND POLARIZATION AND ROTATION OF THE SUBBAND PSEUDOSPIN}

Figure 2 shows the average pseudospin polarization $\left\langle S^{2}\right\rangle$ and its $z$-component $\left\langle S_{z}\right\rangle$ in the ground state. These results are a first indication for a transition from one- to two-component states. Indeed, for narrow quantum wells with small values of $w$, the subband gap is too large to allow for a population of the higher subband $\sigma=\uparrow$, and the system is fully polarized in the $z$-direction - one retrieves the $2 \mathrm{D}$ limit where only the lowest subband is relevant. That is also the case in larger wells with a high bias $V$ that effectively reduces the well width by creating a triangular confinement potential. This needs to be contrasted to $w \sim 7 l_{B}$, where one notices a reduction of the pseudospin polarization (both for $\left\langle S^{2}\right\rangle$ and $\left\langle S_{z}\right\rangle$ ) that indicates an increase in the population of the upper subband. In the black region of Fig. 2, the vanishing values for both quantities are a strong indication for a transition to a pseudospin-singlet state with equal population of both subbands. Notice that in the full parameter range the polarization $\left\langle S_{z}\right\rangle$ never becomes negative such that there is no population inversion between the lowest two subbands. The absence of such population inversion corroborates the validity of the model assumption to restrict the electron dynamics to the lowest two subbands.

$|\langle 331 \mid \mathrm{GS}\rangle|$

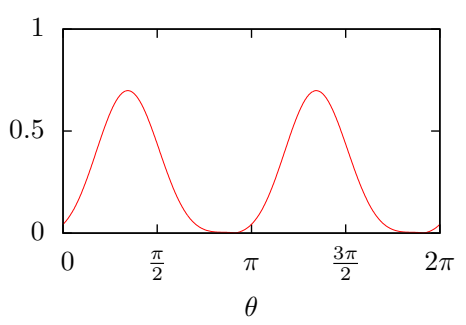

FIG. 3: (Color online) Modulus of the overlap of (331) with the ground state in the lowest LL versus spin-rotation angle of (331), here $w=10, V=1$ and the number of electrons is $N=10$.

In order to interpret the results for the polarization, one needs to realize that, even if the Halperin (331) state has a polarization $S_{z}=0$ in the $z$-direction, it is not a singlet since it is not an eigenstate of the pseudospin $S^{2}$. As a consequence, the (331) state is not invariant under rotations of the pseudospin, and one finds indeed that it possesses an average polarization $\left\langle S^{2}\right\rangle \neq 0$. Under rotation in the appropriate direction, the (331) state may thus be encountered for intermediate pseudospin polarizations, that is for $\left\langle S_{z}\right\rangle \neq 0$ such as in the red region of Fig. 2 that separates the completely polarized system (white) at low $w$ and high $V$ from the (black) singlet region at large values of $w$ and low bias $V$. This rotation towards an appropriate frame of reference may be implemented for the trial wave functions with the help of a variational parameter $\theta$, that allows one to maximize the overlap of the ground state with the (331) state and other possible candidate wave functions. The appropriate transformation on the electron coordinates reads

$$
\left(\begin{array}{c}
z_{i}^{\uparrow} \\
z_{i}^{\downarrow}
\end{array}\right) \longmapsto\left(\begin{array}{cc}
\cos \frac{\theta}{2} & -\sin \frac{\theta}{2} \\
\sin \frac{\theta}{2} & \cos \frac{\theta}{2}
\end{array}\right)\left(\begin{array}{c}
z_{i}^{\uparrow} \\
z_{i}^{\downarrow}
\end{array}\right) .
$$

The strength of this variational approach is illustrated in Fig. 3, where we have depicted the evolution of the overlap of the (331) state with the ground state as a function of $\theta$ for a set of parameters $(w=10$ and $V=1)$ that corresponds precisely to an intermediate pseudospin polarization. Whereas the overlap almost vanishes for $\theta=0$ that is in the absence of a rotation and remains small in the $\pi / 2$-rotated frame used in the study of the symmetric quantum well,, 9 it exceeds $70 \%$ for an optimal angle of $\theta_{\mathrm{opt}} \simeq 1.08$. The $\pi$ periodicity in the angledependence of the overlap reflects the fact that the $z$ component of the pseudospin polarization in the rotated frame vanishes for both $\theta_{\text {opt }}$ and $-\theta_{\text {opt }}$, in contrast to maximally polarized states that show a $2 \pi$ periodicity (results not shown). Notice finally that the rotation of the (331) allows us to obtain a state with partial subband polarization $\left(\left\langle S_{z}\right\rangle \neq 0\right)$ although one maintains $\left\langle S_{z}\right\rangle=0$ in the rotated frame.

\section{RESULTS AND DISCUSSION}

\section{A. Parameter regions of maximal overlap}

The central result of our paper is shown in Fig. 4. where we present the optimal overlap [Fig. 4(a)] between the ground state and the (331) Halperin state at the rotation angle $\theta_{\text {opt }}$ that maximizes the overlap within our variational procedure. We have chosen a magnetic field of $B=14.2 \mathrm{~T}$, such as to make a comparison with recent experimental results 14 To corroborate our findings, we present results for a different value of the magnetic field $(B=7 \mathrm{~T})$ in Sec. IVD. The value of this angle is represented in Fig. 4(b), with respect to the reference angle $\theta_{\mathrm{SQW}}=\pi / 2$ of the symmetric square well with no bias investigated in Ref. 9. One notices that the maximal overlap, which is in the $70 \%$ range, is indeed situated in the arc-shaped region of intermediate pseudospin polarization depicted in Fig. 2 Concerning the black regions in Fig. 4 , where the overlap with the (331) state vanishes, we have found that the singlet state at large $w$ and small 


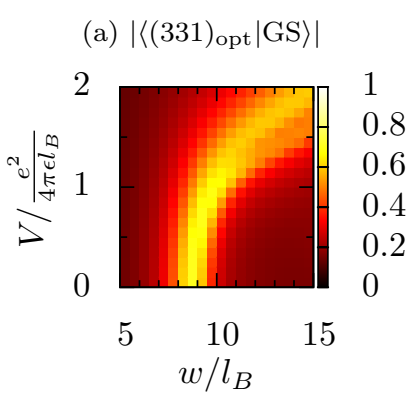

(c) $\left|\left\langle(331)_{\text {pol }} \mid G S\right\rangle\right|$

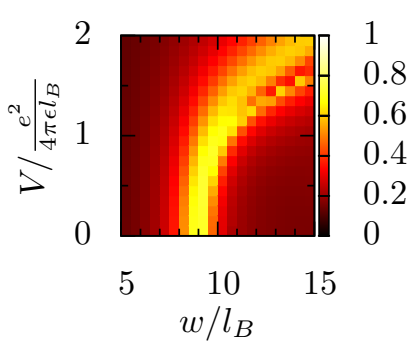

(b) $\theta_{\mathrm{opt}}-\theta_{\mathrm{SQW}}$

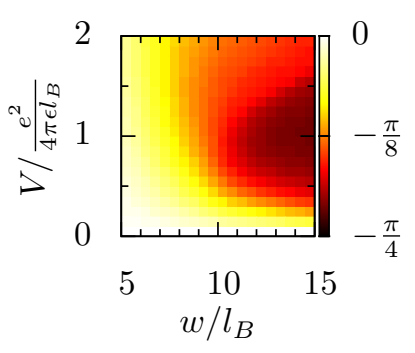

(d) $\theta_{\text {pol }}$

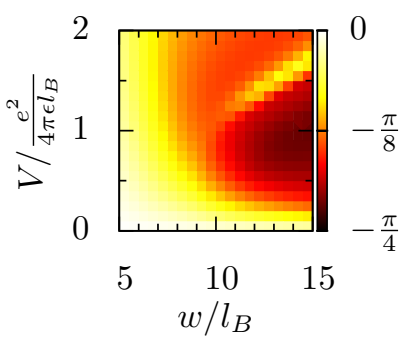

FIG. 4: (Color online) (a) Optimized overlap between the ground and the (331) Halperin state for for $N=10$, $N_{B}=17$ in the lowest LL. (b) Angle that optimizes the above overlap with respect to the angle $\theta_{\mathrm{SQW}}=\pi / 2$, which is the optimal angle in the symmetric square well!9 (c) Overlap between the ground and the (331) state for the mean polarization angle (5), which is depicted in (d).

$V$ has a reasonably high overlap with the compressible Haldane-Rezayi state, ${ }^{[19}$ whereas in the small-w/large- $V$ limit the Pfaffian $\underline{4}$ has the largest overlap (results not shown). However, we stress that in this limit of the lowest LL the (incompressible) Pfaffian is not the true ground state, but rather the (compressible) composite-fermion Fermi liquid. Even if a direct comparison between these states is delicate in the spherical geometry, because the states do not occur at the same shift, we have checked that the ground state above the arc-shaped region in Fig. 4(a) (at $N=10$ and $N_{B}=18$, i.e. for a shift $\delta=-2$ ) is adiabatically connected to the composite-fermion Fermi liquid at $w=V=0$. The incompressible Halperin state is thus surrounded by compressible states, and the FQHE can therefore be induced by increasing the bias for the appropriate well width.

\section{B. Optimal pseudospin angle}

In order to obtain a better insight into the meaning of the angle $\theta(w, V)$ that optimizes the overlap, we consider again the pseudospin polarization of the ground state. Its average direction is characterized by the angle

$$
\theta_{\mathrm{pol}}=\arctan \left(\frac{\left\langle S_{x}\right\rangle}{\left\langle S_{z}\right\rangle}\right)
$$

which only depends on the $x$ - and the $z$-component of the polarization because the $y$-component vanishes, $\left\langle S_{y}\right\rangle=0$. This is a consequence of the considered rotation (4), which only concerns the azimuthal angle $\theta$, and we have tested numerically that $\left\langle S^{2}\right\rangle=\left\langle S_{z}^{2}\right\rangle+\left\langle S_{y}^{2}\right\rangle$ remains invariant under this transformation. The polarization angle $\theta_{\text {pol }}$ is shown in Fig. 4(d) as a function of the parameters $w$ and $V$, and one notices an excellent agreement with the angle that optimizes the overlap between the ground and the (331) state in Fig. 4(b). Furthermore, we have depicted in Fig. 4(c) the same overlap for the polarization angle $\theta_{\text {pol }}$ instead of the optimal angle, and one finds that the difference between the overlaps is incremental in the region of interest. Our results therefore indicate that, in order to investigate the (331) Halperin state in a BQW, one needs to use a rotated basis that is determined by the polarization angle of the sublevel pseudospin.

\section{Energy gaps}

We now turn to the study of neutral energy gaps that is the minimal gap between the $L=0$ ground state and the first excited state (generally at $L \neq 0$ ) at fixed particle and flux number. In the case of Laughlin states, 1 this gap corresponds to the so-called magnetoroton minimum.20 Although this is not the activation gap obtained in magneto-transport measurements, the neutral gaps provide a measure of the ground-state stability. The neutral energy gaps are presented in Fig. $5 \mathrm{a}$, in comparison with the overlap of the (331) state with the ground state [Fig. 5b, same as Fig. 4a, for the optimal pseudospin-rotation angle $\theta$ of the former. As in the previous discussion, we show results for $N=10$ particles and $N_{B}=17$ flux quanta.

The gaps are largest $\left(\sim 0.02 \ldots 0.035 e^{2} / 4 \pi \epsilon l_{B}\right)$ in the upper-left part of the region of highest overlap $(75 \%)$. Our results for the neutral gaps therefore corroborate the overall picture that the (331) state is stable in the parameter range where one finds the largest overlap with the ground state obtained within exact diagonalization. Interestingly, one obtains a rather abrupt decrease of the gap below the arc-shaped region of maximal gap, while it remains substantial $\left(\sim 0.01 e^{2} / 4 \pi \epsilon l_{B}\right)$ in the region above the gap, in which one expects a polarized state. Whereas the origin of this decrease remains to be understood, as well as its relative increase again at lower values of $V$ (and larger values of $w$ ), one notices that it is anticipated by the overlaps (Fig. 5b where one finds a qualitatively similar fine structure in the arc-shaped region of the parameter space. 


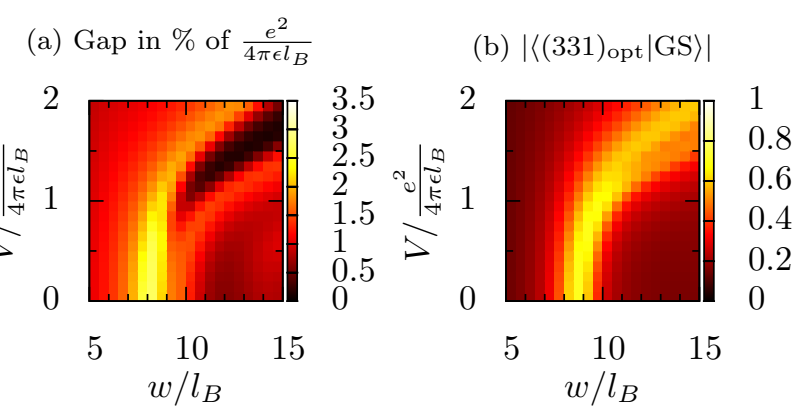

FIG. 5: (Color online) (a) Neutral energy gap [in \% of the Coulomb energy $\left.e^{2} /\left(4 \pi \epsilon l_{B}\right)\right]$ and (b) optimized overlap of (331) with the ground state for $N=10$ and

$$
N_{B}=17 \text {. }
$$

\section{Results for a different value of the magnetic field}

In addition to a magnetic field $B=14.2 \mathrm{~T}$, a value used in the previous subsections, we present here results for a different value $B=7 \mathrm{~T}$ in order to test the global picture presented above. Figure 6 represents the same quantities for this value (for $N=10$ particles and $N_{B}=17$ flux quanta) as Fig. 4 for $B=14.2 \mathrm{~T}$. (a) $\left|\left\langle(331)_{\text {opt }} \mid G S\right\rangle\right|$

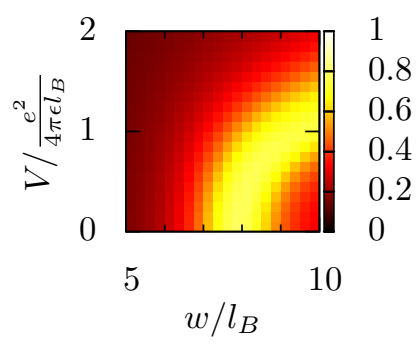

(c) $\left|\left\langle(331)_{\text {pol }} \mid \mathrm{GS}\right\rangle\right|$

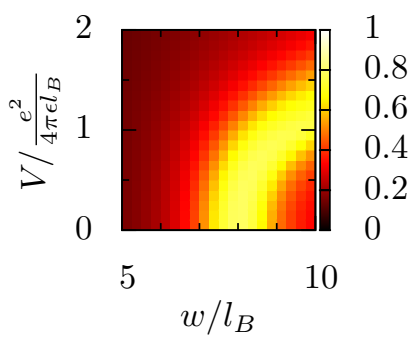

(b) $\theta_{\mathrm{opt}}-\theta_{\mathrm{SQW}}$

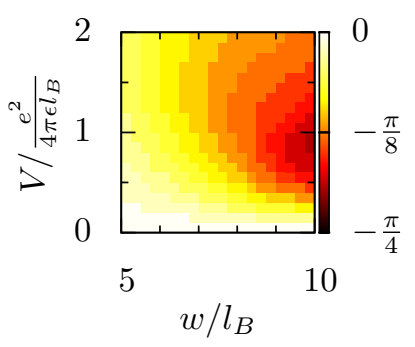

(d) $\theta_{\mathrm{pol}}$

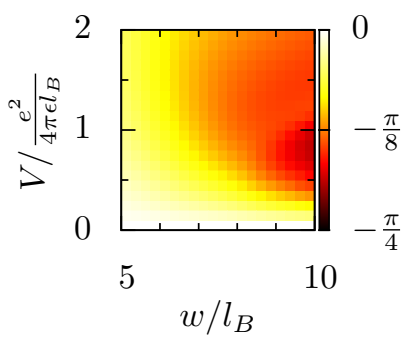

FIG. 6: (Color online) Same quantities as Fig. 4, for a different value of the magnetic field, $B=7 \mathrm{~T}$.

One notices the similarity between the results for $B=$
14.2 $\mathrm{T}$ and $B=7 \mathrm{~T}$, are similar. For both values of the magnetic field the ground state of the system has a large overlap with the (331) state in an arc-shaped region of the parameter space. Quantitatively, two differences are worth pointing out. First, the region of high overlap mentioned above is shifted to slightly lower values of the width for $B=7 \mathrm{~T}$ as compared to $B=14.2 \mathrm{~T}$. This shift as a function of the magnetic field may be understood qualitatively from simple scaling arguments. Consider the $V=0$ limit in which case the (331) state is stabilized when the gain in correlation energy $\sim\left(e^{2} / \epsilon l_{B}\right) \times\left(w / l_{B}\right)$ from filling the higher subband roughly cancels the subband gap $\Delta=\Delta(w, V=0), \frac{9}{9}$ i.e. in our units

$$
\frac{\Delta}{e^{2} / \epsilon l_{B}}=c \frac{a_{B}}{l_{B}} \times\left(\frac{l_{B}}{w}\right)^{2} \sim \frac{w}{l_{B}},
$$

where $c$ is a numerical prefactor and $a_{B}=\hbar^{2} \epsilon / m e^{2}$ is the effective Bohr radius in terms of the electronic band mass $m$. This leads to a $B$-field scaling

$$
\frac{w}{l_{B}} \sim\left(\frac{a_{B}}{l_{B}}\right)^{1 / 3} \propto B^{1 / 6}
$$

for the characteristic parameter at which the (331) state is stabilized. In the present case (Fig. 6), the magnetic field is half of that discussed previously in Fig. 4, such that one expects for the characteristic width parameter $\left(w / l_{B}\right)[B=7 \mathrm{~T}] \simeq 0.9\left(\mathrm{w} / \mathrm{l}_{\mathrm{B}}\right)[\mathrm{B}=14 \mathrm{~T}]$, in agreement with our numerical findings.

As for the second quantitative difference between the two values of the magnetic field, one notices that the overlap of the ground state and the (331) state is slightly enhanced for $B=7 \mathrm{~T}$. It is on the order of $80 \%$ in the high overlap region whereas it is $\sim 70 \%$ for $B=14.2 \mathrm{~T}$.

\section{E. Comparison with experiments}

Notice finally that our variational approach provides an excellent understanding of recent magnetotransport measurements by Shabani et al., who investigated quantum-Hall states in quantum wells with a potential bias applied between the sides ${ }^{[14 \mid 15}$ In the case of the 55-nm wide sample, which we discuss as a representative example here, half-filling of the lowest LL is encountered at a magnetic field of $14.2 \mathrm{~T}$, which is precisely the value we have chosen in our numerical studies. It corresponds to a ratio of $w / l_{B} \simeq 8$ between the well width and the magnetic length. A FQHE could be stabilized for values of the bias around $V=16.5 \mathrm{meV}$ that correspond to $V /\left(e^{2} / 4 \pi \epsilon l_{B}\right) \simeq 1$ in our units. Our overlap calculations in Fig. 4 indicate a similar window for the observation of a FQHE slightly below $w \simeq 10 l_{B}$ that is some $10-20 \%$ larger than the experimental value. Apart from the finite size of the system studied numerically, this slight discrepancy is likely to be due to our BQW model that considers infinite potential barriers, whereas the potential barriers 
are finite $(\sim 200 \mathrm{meV})$ in a realistic situation such that the wave functions $\varphi_{\sigma}(z)$ can penetrate into the barriers. The wave function in the $z$-direction is therefore slightly larger than in the BQW model, where the width of the wave function coincides with the well width $w$. To compensate this effect, one therefore needs to consider a larger effective width within our model.

\section{CONCLUSIONS}

In conclusion, we have presented numerical evidence that a FQHE in the form of a (331) Halperin state can be stabilized in a wide quantum well with a potential bias in an intermediate range, as observed experimentally. From a technical point of view, we have established a model (BQW) that allows one to obtain the wave func- tions, which are associated with the confinement potential. They are the basic ingredient in the calculation of the effective interaction potential, which is used in our exact-diagonalization studies on the sphere. This model may be useful in further studies of the FQHE in wide anisotropic quantum wells. Furthermore, we have shown that the wave-function overlap between the ground state and trial wave functions, as the (331) state here, can be largely enhanced if the state is prepared in a rotated frame that corresponds to the orientation of the sublevelpseudospin polarization. We expect that this rotated frame is also essential in the understanding of other possible FQHE states in the BQW, beyond the (331) state.

We thank Gilles Montambaux for an optimized initial guess of BQW eigenergies. Furthermore, we acknowledge fruitful discussions with Paul Soulé and Zlatko Papić. N.R. was supported by a Keck grant.
1 R. B. Laughlin, Phys. Rev. Lett. 50, 1395 (May 1983), http://link.aps.org/doi/10.1103/PhysRevLett.50. 1395

2 J. K. Jain, Phys. Rev. Lett. 63, 199 (Jul 1989), http: //link.aps.org/doi/10.1103/PhysRevLett.63.199

3 J. Jain, Composite Fermions (Cambridge University Press, 2007)

${ }^{4}$ G. Moore and N. Read, Nuclear Physics B 360, 362 (1991)

${ }^{5}$ B. I. Halperin, Helv. Phys. Acta 56, 75 (1983)

${ }^{6}$ J. P. Eisenstein, G. S. Boebinger, L. N. Pfeiffer, K. W. West, and S. He, Phys. Rev. Lett. 68, 1383 (Mar 1992), http://link.aps.org/doi/10.1103/ PhysRevLett.68.1383

K. Moon, H. Mori, K. Yang, S. M. Girvin, A. H. MacDonald, L. Zheng, D. Yoshioka, and S.-C. Zhang, Phys. Rev. B 51, 5138 (Feb 1995), http://link.aps.org/doi/ 10.1103/PhysRevB.51.5138

$\checkmark$ M. O. Goerbig, Rev. Mod. Phys. 83, 1193 (Nov 2011), http://link.aps.org/doi/10.1103/RevModPhys. 83.1193

9 Z. Papić, G. Möller, M. V. Milovanović, N. Regnault, and M. O. Goerbig, Phys. Rev. B 79, 245325 (Jun 2009), http: //link.aps.org/doi/10.1103/PhysRevB.79.245325

IU M. R. Peterson, Z. Papić, and S. Das Sarma, Phys. Rev. B 82, 235312 (Dec 2010), http://link.aps.org/doi/10. 1103/PhysRevB.82.235312

${ }^{11}$ V. W. Scarola, C. May, M. R. Peterson, and M. Troyer, Phys. Rev. B 82, 121304 (Sep 2010), http://link.aps. org/doi/10.1103/PhysRevB.82.121304
12 D. R. Luhman, W. Pan, D. C. Tsui, L. N. Pfeiffer, K. W. Baldwin, and K. W. West, Phys. Rev. Lett. 101, 266804 (Dec 2008), http://link.aps.org/doi/10.1103/ PhysRevLett.101.266804

15 J. Shabani, T. Gokmen, and M. Shayegan, Phys. Rev. Lett. 103, 046805 (Jul 2009), http://link.aps.org/doi/ 10.1103/PhysRevLett.103.046805

${ }^{14}$ J. Shabani, T. Gokmen, Y. T. Chiu, and M. Shayegan, Phys. Rev. Lett. 103, 256802 (Dec 2009), http://link. aps.org/doi/10.1103/PhysRevLett.103.256802

15 J. Shabani, Y. Liu, M. Shayegan, L. N. Pfeiffer, K. W. West, and K. W. Baldwin, ArXiv e-prints(Jun. 2013), arXiv:1306.5290 [cond-mat.mes-hall]

16 M. O. Goerbig and N. Regnault, Phys. Rev. B 75, 241405 (Jun 2007), http://link.aps.org/doi/10.1103/ PhysRevB.75.241405

If Z. Papić, M. O. Goerbig, and N. Regnault, Phys. Rev. Lett. 105, 176802 (Oct 2010), http://link.aps.org/doi/ 10.1103/PhysRevLett.105.176802

18 R. de Gail, N. Regnault, and M. O. Goerbig, Phys. Rev. B 77, 165310 (Apr 2008), http://link.aps.org/doi/10. 1103/PhysRevB.77.165310

${ }^{19}$ F. D. M. Haldane and E. H. Rezayi, Phys. Rev. Lett. 60, 956 (Mar 1988), http://link.aps.org/doi/10.1103/ PhysRevLett.60.956

20 S. M. Girvin, A. H. MacDonald, and P. M. Platzman, Phys. Rev. B 33, 2481 (Feb 1986), http://link.aps.org/doi/ 10.1103/PhysRevB.33.2481 